\title{
2-dimensional models of rapidly rotating stars
}

\section{Hydrostatic and acoustic models with $\Omega=\Omega(r, \theta)$}

\author{
I. W. Roxburgh ${ }^{1,2}$ \\ 1 Astronomy Unit, Queen Mary, University of London, Mile End Road, London E1 4NS, UK \\ e-mail: I.W. Roxburgh@qmul . ac . uk \\ 2 LESIA, Observatoire de Paris, Place Jules Janssen, 92195 Meudon Cedex, France
}

Received 28 February 2006 / Accepted 23 April 2006

ABSTRACT

\begin{abstract}
Aims. We show how to construct 2-dimensional models of rapidly rotating stars in hydrostatic equilibrium for any $\Omega(r, \theta)$, given the density $\rho_{\mathrm{m}}(r)$ along any one angle $\theta_{\mathrm{m}}$. If the hydrogen abundance $X_{\mathrm{m}}(r)$ is given on $\theta_{\mathrm{m}}$ then the adiabatic exponent $\Gamma_{1}(r, \theta)$ can by determined, yielding a self consistent acoustic model that can be used to investigate the oscillation properties of rapidly rotating stars. Methods. The system of equations governing the hydrostatic structure is solved by iteration using the method of characteristics and spectral expansion, subject to the condition that $\rho(r, \theta)=\rho_{\mathrm{m}}(r)$ on $\theta=\theta_{\mathrm{m}} . \Gamma_{1}(r, \theta)$ is calculated from the equation of state under the assumption that $X\left(r, \theta_{\mathrm{m}}\right)=X_{\mathrm{m}}(r)$ and is constant on surfaces of constant entropy. Alternatively $\Gamma_{1}$ can be approximated by taking $X$ constant in the equation of state and equal to the surface value.

Results. Results are presented for an evolved main sequence star of $2 M_{\odot}$ with the angular velocity a function only of radius $\Omega=\Omega(r)$, evolved to a central hydrogen abundance of $X_{\mathrm{c}}=0.35$. The model is first calculated using a spherically averaged stellar evolution code, where the averaged centrifugal force $2 \Omega^{2} r / 3$ is added to gravity. The resulting $\rho_{\mathrm{m}}(r), X_{\mathrm{m}}(r)$ are then used as input to determine the 2-dimensional model.

Conclusions. The procedure described here gives self consistent hydrostatic and acoustic models of rapidly rotating stars for any $\Omega(r, \theta)$.
\end{abstract}

Key words. stars: rotation - stars: evolution

\section{Introduction}

One of the major problems inhibiting the use of stellar oscillations to diagnose the internal structure of stars, and thereby to test and develop our understanding of stellar evolution, is the effect of rapid rotation. Recent work by Lignières et al. (2006) and Reese et al. (2006) on the oscillation of polytropic models of uniformly rotating stars, has shown that perturbation analysis breaks down and full 2-dimensional studies are needed for moderate rotation velocities $\left(v \sim 50 \mathrm{~km} \mathrm{~s}^{-1}\right)$, and that a major effect on the oscillation frequencies is the geometrical (and acoustical) oblateness of the star. Most stars of spectral type $F$ and earlier have rotation velocities in excess of $50 \mathrm{~km} \mathrm{~s}^{-1}$ so we need to have realistic 2-dimensional models of rotating stars to analyse their oscillation properties, and to develop techniques to utilise the data from high precision asteroseismology missions such as COROT (Baglin et al. 2002), to map, or constrain, models of stellar structure and evolution.

In a previous paper (Roxburgh 2004) we presented selfconsistent 2-dimensional models of homogeneous main sequence stars where the angular velocity $\Omega=$ const. But to interpret and use observational data on the oscillations of stars we need realistic 2-dimensional models of rapidly rotating stars with different rotation profiles $\Omega(r, \theta)$, and in different stages of evolution. By studying the oscillation properties of such models we can hope to interpret observational data and develop techniques that will enable us to use such data to diagnose the internal structure and dynamics of rotating stars. We here present an algorithm to construct such models.
The adiabatic oscillations of a spherical star are governed solely by the acoustic structure of the star, that is the hydrostatic structure: density $\rho(r)$, pressure $P(r)$ and gravity $g(r)$; together with the adiabatic exponent $\Gamma_{1}(r)$. The oscillation properties do not depend on whether or not the star is in thermal equilibrium, only that it is in hydrostatic equilibrium with known $\Gamma_{1}(r)$. The hydrostatic structure of a star is completely determined by the density density profile $\rho(r)$ and surface value of the pressure $P_{\mathrm{s}}$; $g(r)$ is given by Poisson's equation and $P(r)$ by hydrostatic support. The interior structure is insensitive to the value of $P_{\mathrm{s}}$. The adiabatic exponent $\Gamma_{1}$ can be determined from $P(r), \rho(r)$ and the composition $X_{j}(r)$ through the equation of state; for hydrogen burning stars it is sufficient to have just the hydrogen abundance $X(r)$. Note that in the fully ionised interior of most stars $\Gamma_{1} \approx 5 / 3$ for any $X$, whereas in the outer layers $X$ retains its initial value, so $\Gamma_{1}(r)$ could be adequately approximated by taking the surface value of $X$ in the equation of state for all $P, \rho$. For stars other than the Sun we can only expect to detect oscillations of modes of low degree, which have very similar properties in the outer layers of a star, so we can only hope to probe the interior structure of a star with such data; taking $\Gamma_{1}=5 / 3$ is then often adequate for the development of diagnostic and inversion techniques that constrain or map the internal hydrostatic structure of stars (cf. Roxburgh \& Vorontsov 2003).

As shown below a similar situation applies to rotating stars; the 2-dimensional hydrostatic structure $\rho(r, \theta), P(r, \theta)$ and gravitational potential $\Phi(r, \theta)$ are determined solely by the angular velocity $\Omega(r, \theta)$, the density $\rho_{\mathrm{m}}(r)=\rho\left(r, \theta_{\mathrm{m}}\right)$ and the surface pressure $P_{\mathrm{s}}$ along any one angle $\theta_{\mathrm{m}}$. The adiabatic exponent 
$\Gamma_{1}(r, \theta)$ is determined from $P(r, \theta), \rho(r, \theta)$ and the 2-dimensional hydrogen profile $X(r, \theta)$. If we have some model of the mixing processes inside a rotating star, e.g. $X$ constant on surfaces of constant entropy, then it is sufficient to have $X_{\mathrm{m}}(r)$ along $\theta_{\mathrm{m}}$. Again reasonable values of $\Gamma_{1}$ can be estimated from $P, \rho$ and the surface value of $X$. Again there is no requirement that thermal equilibrium be satisfied. Acoustic models of rapidly rotating stars with any $\Omega(r, \theta)$ can therefore be generated by prescribing the radial density and hydrogen profiles $\rho_{\mathrm{m}}(r), X_{\mathrm{m}}(r)$ along an angle $\theta_{\mathrm{m}}$, and the oscillation properties of such models investigated to advance our understanding of the effects of rotation on oscillations, and to develop diagnostic and inversion techniques for use with observational data. For such models to be realistic the input values $\rho_{\mathrm{m}}(r), X_{\mathrm{m}}(r)$ should, at least approximately, correspond to our expectations as to the run of these variables in models of evolved stars.

Stellar evolutionary models that include rotation are almost always spherically averaged models where the angular velocity is taken to vary with radius $\Omega=\Omega(r)$ (cf. Maeder 2003). The argument for such shellular rotation being that, as there is no stabilising effect of buoyancy on surfaces of constant entropy, horizontal mixing is efficient in maintaining $\Omega$ and the chemical composition constant on such surfaces (cf. Zahn 1992). In these models the centrifugal force averaged over a sphere, $2 \Omega^{2} r / 3$, is added to gravity, and mild turbulence, generated by shear and circulation currents, contributes to thermal balance, produces some mixing of chemical composition and maintains the $\Omega$ profile, Such models give the spherically averaged values of density $\rho_{\mathrm{m}}(r)$, and hydrogen abundance $X_{\mathrm{m}}(r)$. With $\Omega=\Omega(r)$ perturbation analysis gives these averaged values as those along the root of the second Legendre polynomial $\theta_{\mathrm{m}}=\cos ^{-1}(1 / \sqrt{3})$ (cf. Roxburgh 1964; Faulkner et al. 1968).

We can extend such spherically averaged models to give 2-dimensional hydrostatic models by requiring that the 2-d model has the 1-d $\rho_{\mathrm{m}}(r)$ along the angle $\theta_{\mathrm{m}}$. Thermal balance does not enter into this calculation but is implicitly assumed to be maintained by mild turbulence and circulation currents as in the spherically averaged model. If further $X(r, \theta)$ is taken to be constant along surfaces of constant entropy then, through the equation of state, $P(r, \theta), \rho(r, \theta)$ and $X_{\mathrm{m}}(r)$ give $X(r, \theta)$ and $\Gamma_{1}(r, \theta)$. Hence given a spherically averaged model of an evolved star we can construct self consistent 2-dimensional hydrostatic and acoustic models of rapidly rotating stars which can be used for studying their oscillation properties.

\section{The hydrostatic structure for $\Omega=\Omega(r, \theta)$}

The equations governing the hydrostatic structure of a rotating star are

$$
\begin{aligned}
\frac{\nabla P}{\rho} & =-\nabla \Phi+\Omega^{2} \varpi \\
\nabla^{2} \Phi & =4 \pi G \rho
\end{aligned}
$$

where $P(r, \theta), \rho(r, \theta), \Phi(r, \theta)$ are the pressure, density and gravitational potential, $\Omega(r, \theta)$ is the angular velocity, $(r, \theta, \phi)$ are spherical polar coordinates and $\varpi=(r \sin \theta, r \cos \theta, 0)$ is the vectorial distance from the rotation axis. The inertia of any circulation or turbulence, and viscous effects, are neglected.

Eliminating $P$ by taking the curl (rot) of Eq. (1) gives

$\operatorname{curl}\left(\rho\left[\nabla \Phi-\Omega^{2} \varpi\right]\right)=0$.

Equations (2) and (3) constitute a 3rd order boundary value problem which determines $\rho(r, \theta)$ given the value of $\rho\left(r, \theta_{\mathrm{m}}\right)=\rho_{\mathrm{m}}(r)$ on one angle $\theta_{\mathrm{m}}$, and that $\Phi$ matches onto a solution of Laplace's equation outside the star.

The pressure $P(r, \theta)$ is then given by Eq. (1) subject to a surface boundary condition on $P$. Note that the value of $P$ along $\theta_{\mathrm{m}}$ will not be exactly the same as the value in the spherically averaged model since the 2 -dimensional value of the gravitational field along $\theta_{\mathrm{m}}$ will not be exactly the same as that in the spherically averaged model, nor will the mass of the distorted model be exactly the same as that of the spherical input model; the differences come from neglecting the distortion factors in rotating stars in the spherically averaged model (cf. Roxburgh 2004). One could scale the density of the input model to give the same total mass if desired.

For slow rotation we can eliminate $\rho$ between Eqs. (2) and (3) and solve the resulting 3rd order system by perturbation analysis (cf. Roxburgh 2001). For rapid rotation we solve the equations by successive iteration as follows:

1. take as an initial guess $\rho(r, \theta)=\rho_{\mathrm{m}}(r)$;

2. solve Poisson's Eq. (2) for $\Phi(r, \theta)$ given $\rho(r, \theta)$;

3. solve Eq. (3) for $\rho(r, \theta)$ given $\Phi(r, \theta)$ subject to the condition $\rho\left(r, \theta_{\mathrm{m}}\right)=\rho_{\mathrm{m}}(r)$ the input density on $\theta_{\mathrm{m}} ;$

4. goto 2 and repeat until the solution has converged.

For a given $\Phi$ Eq. (3) is a first order linear PDE which can be solved by the method of characteristics; for given $\rho$ Eq. (2) is second order linear and can be solved by spectral analysis.

\section{Details of the solution}

The solution is represented by the values of $\rho_{i j}, P_{i j}, \Phi_{i j}$ on a spherical polar coordinate mesh $\left(r_{i}, \theta_{j}\right)$ where $i=0, N_{i}$, and $j=0, N_{j}$. The radial mesh of the input model is $i=0, N$ and $i=N+1, N_{i}$ is an extension of this mesh into the surrounding space, large enough to contain all regions of the 2-dimensional model. The $\theta_{j}$ are uniformly distributed, $\theta_{j}=(\pi / 2)\left(j / N_{j}\right)$. For convenience the angle $\theta_{\mathrm{m}}$ on which the input model is given is taken to be on the mesh $\theta_{j}$.

\subsection{Solution for $\rho$ given $\Phi$}

\section{Defining}

$\boldsymbol{F}=\nabla \Phi-\Omega^{2} \varpi$

we can write Eq. (3) as

$F_{\theta} \frac{\partial \rho}{\partial r}-F_{r} \frac{1}{r} \frac{\partial \rho}{\partial \theta}=-\rho \operatorname{curl}(\boldsymbol{F})=\rho \operatorname{curl}\left(\Omega^{2} \varpi\right)$.

The characteristics are the curves $r=r(\theta)$ given by

$\frac{\mathrm{d} r}{\mathrm{~d} \theta}=-r \frac{F_{\theta}}{F_{r}}=-\frac{\left(\partial \Phi / \partial \theta-\Omega^{2} r^{2} \sin \theta \cos \theta\right)}{\left(\partial \Phi / \partial r-\Omega^{2} r \sin ^{2} \theta\right)}$

and along the characteristics $r=r(\theta), \rho$ is given by

$$
\frac{1}{\rho} \frac{\mathrm{d} \rho}{\mathrm{d} \theta}=-r \frac{\operatorname{curl}\left(\Omega^{2} \varpi\right)}{\left(\partial \Phi / \partial r-\Omega^{2} r \sin ^{2} \theta\right)} .
$$

Describing the characteristic through the point $r_{\mathrm{m}}$ on the reference angle $\theta_{\mathrm{m}}$ as $r=r_{\mathrm{m}} g_{\mathrm{m}}(\theta)$, then $g_{\mathrm{m}}$ satisfies the ordinary differential equation

$$
\frac{\mathrm{d} g_{\mathrm{m}}}{\mathrm{d} \theta}=-\frac{1}{r_{\mathrm{m}}} \frac{\left(\partial \Phi / \partial \theta-\Omega^{2} r^{2} \sin \theta \cos \theta\right)}{\left(\partial \Phi / \partial r-\Omega^{2} r \sin ^{2} \theta\right)}
$$


With $\Phi, \partial \Phi / \partial r, \partial \Phi / \partial \theta$ known on the mesh $\left(r_{i}, \theta_{j}\right)$ we integrate Eq. (8) on the mesh $j$, interpolating in $r$ on this mesh for values of $\partial \Phi / \partial r, \partial \Phi / \partial \theta$ at $r=r_{\mathrm{m}} g(\theta)$. The boundary condition is that $g_{\mathrm{m}}=1$ at $\left(r_{\mathrm{m}}, \theta_{\mathrm{m}}\right)$. This gives the characteristics of Eq. (3).

Since

$\operatorname{curl}\left(\Omega^{2} \varpi\right)=\frac{1}{r}\left[\frac{\partial}{\partial r}\left(\Omega^{2} r^{2} \sin \theta \cos \theta\right)-\frac{\partial}{\partial \theta}\left(\Omega^{2} r \sin ^{2} \theta\right)\right]$

Eq. (7) can be integrated along the characteristics $r=r_{\mathrm{m}} g_{\mathrm{m}}(\theta)$, to give $\rho$ along these characteristics. The solution can then be transferred onto the mesh $\left(r_{i}, \theta_{j}\right)$ by cubic interpolation.

\subsection{Solution for $\Phi$ given $\rho$}

Given $\rho(r, \theta)$ on the reference mesh $\left(r_{i}, \theta_{j}\right)$ we solve the Poisson Eq. (2) for $\Phi$ using the algorithm described in Roxburgh (2004). $\rho$ and $\Phi$ are represented by finite sums of Legendre polynomials

$\rho(r, \theta)=\sum_{0}^{N_{k}} c_{k}(r) P_{2 k}(\cos \theta)$

$\Phi(r, \theta)=\sum_{0}^{N_{k}} f_{k}(r) P_{2 k}(\cos \theta)$

where the $f_{k}$ satisfy the equations

$\frac{1}{r^{2}} \frac{\mathrm{d}}{\mathrm{d} r}\left(r^{2} \frac{\mathrm{d} f_{k}}{\mathrm{~d} r}\right)-\frac{2 k(2 k+1)}{r^{2}} f_{k}=4 \pi G c_{k}$

subject to the boundary conditions $f_{k}(0)=0, k \neq 0$, and

$(2 k+1) f_{k}+r \frac{\mathrm{d} f_{k}}{\mathrm{~d} r}=0$ at $r=R_{\mathrm{s}}$

where $R_{\mathrm{s}}=r\left(N_{i}\right)$ is a spherical surface outside the star.

The decomposition of the density was done algebraically by simply demanding that (at each radius $r_{i}$ ) Eqs. (10) be satisfied at the angles $\theta_{k}, k=0, N_{k}$, (where the $\theta_{k}$ are a subset of $\theta_{j}$ ), and solving the resulting matrix equations to give

$c_{k}=W_{k n}^{-1} \rho_{n}, \quad W_{k n}=P_{2 k}\left(\cos \theta_{n}\right), k, n=0, N_{k}$.

This simple algorithm worked remarkably well, much better than seeking to determine the $c_{k}$ by integrating $\rho(r, \theta) P_{2 k}(\cos \theta)$ over sphere using a much finer mesh in angle.

Having determined the $c_{k}$ the coefficients $f_{k}$ were determined from an integral representation of the solution of Eqs. (12), (13) namely

$f_{k}=r^{2 k} \int_{R_{\mathrm{s}}}^{r} \frac{4 \pi G}{r^{4 k+2}}\left[\int_{0}^{r} c_{k}(r) r^{2 k+2} \mathrm{~d} r\right] \mathrm{d} r-\lambda_{k} r^{2 k}$

where

$\lambda_{k}=\frac{4 \pi G}{(4 k+1) R_{\mathrm{s}}^{4 k+1}} \int_{0}^{R_{\mathrm{s}}} c_{k}(r) r^{2 k+2} \mathrm{~d} r$.

Provided one sets $c_{k}=0$ when it is sufficiently small (e.g. $\left.\left|c_{k}\right|<10^{-10} \rho_{\mathrm{c}}\right)$ this simple algorithm works well. Detailed tests of this and the density decomposition algorithm were reported in Paper I (Roxburgh 2004).

\subsection{Solution for $P$ given $\rho$ and $\Phi$}

The characteristics of Eq. (3) are simply the surfaces $P=$ const. This can be seen from the fact that Eq. (6) is simply $\mathrm{d} \boldsymbol{r} \cdot \boldsymbol{F}=0$ which from Eq. (1) gives $\mathrm{d} \boldsymbol{r} \cdot \nabla P=0$ so that the characteristics are orthogonal to $\nabla P$ and hence along surface $P=$ const.

Since we have already determined the characteristics $r=$ $r_{\mathrm{m}} g_{\mathrm{m}}(\theta)$ all we need is the value of $P$ along a radius vector at any one angle $\theta, P(r, \theta)$ is then constant along the characteristic. Taking the angle as $\theta_{\mathrm{m}}$ we therefore integrate

$\frac{\partial P}{\partial r}=-\rho\left(\frac{\partial \Phi}{\partial r}-\Omega^{2} r \sin ^{2} \theta_{\mathrm{m}}\right)$

subject to the surface boundary condition that $P\left(R_{\mathrm{o}}, \theta_{\mathrm{m}}\right)$ is equal to the value in the input model. As mentioned above the resulting values for $P\left(r, \theta_{\mathrm{m}}\right)$ will not be exactly the same as the values in the input model, since $\partial \Phi / \partial r$ for the converged model will not be exactly the same as that in the input model.

\subsection{Iteration to convergence}

To start the iteration we take the initial guess at the density $\rho(r, \theta)$ as the spherically symmetric density $\rho_{\mathrm{m}}(r)$ of the spherically averaged input model, setting $\rho=0 r>R_{\mathrm{o}}$ where $R_{\mathrm{O}}=r(N)$ is the radius of the input model. We then successively repeat the calculations described in Sects. 3.1 and 3.2 until the solution has converged - convergence being defined as when the fractional change in polar and equatorial radii between successive iterations is less $10^{-9}$.

\subsection{Structure of the surface layers}

In general, for arbitrary $\Omega$, the surfaces $P=$ const. and $\rho=$ const. do not coincide. Hence the solution can have $\rho=0$ on surfaces $P>0$. For the solution to be well behaved at the surface we need $\operatorname{curl}\left(\Omega^{2} \varpi\right)=0$, or $\Omega=\Omega(\varpi)$ in the surface layers ( $\varpi=r \sin \theta$ ), so that $\rho$ is constant on surfaces of constant $P$. We force this to be the case in the example given below by taking $\Omega=$ const. in the outer layers of the star.

The structure of the surface layers of a rotating star is a problem still waiting for a solution. Meridional circulation driven by the rotation can become very large in regions of low density and pressure, so that neglect of the momentum of such circulation in the hydrostatic condition may become invalid; moreover the circulation may be shear unstable producing small scale turbulence. The time scale of the circulation is very small in these layers so that transport of angular momentum is rapid and we may anticipate that the system reaches a steady state. One possible steady state is where there is no circulation and the rotation departs only very slightly from a constant value (cf. Roxburgh 1964; Osaki 1966), so that taking $\Omega=$ const. in the outer layers is a reasonable approximation.

\section{Acoustic models}

In order to use such models in studies of the oscillation of rapidly rotating stars we also need the value of the adiabatic exponent $\Gamma_{1}(r, \theta)$. Since $P(r, \theta), \rho(r, \theta)$ are known, if we knew the 2-dimensional distribution of chemical composition $X(r, \theta)$ then $\Gamma_{1}$ could be determined from the equation of state. If all we are given is the distribution of $X_{\mathrm{m}}(r)$ along the input radius at $\theta_{\mathrm{m}}$, and we assume that $X$ is constant on surfaces of constant entropy, we 


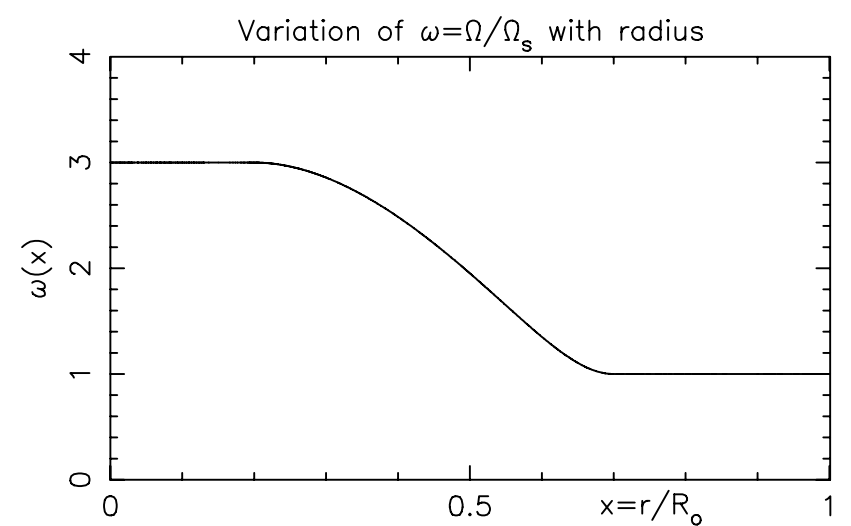

Fig. 1. Variation of $\omega=\Omega / \Omega_{\mathrm{s}}$ with radius in the spherically averaged evolved model of a $2 M_{\odot}$ star.

can determine the equation of adiabats $r=r_{\mathrm{m}} h(\theta)$, along which $X$ is constant, by solving the equations $\left(\nabla P-\Gamma_{1} \nabla \rho\right) \cdot \mathrm{d} \boldsymbol{r}=0$ or

$\frac{\mathrm{d} h}{\mathrm{~d} \theta}=-\frac{1}{r_{\mathrm{m}}} \frac{\left(\partial P / \partial \theta-\Gamma_{1} \partial \rho / \partial \theta\right)}{\left(\partial P / \partial r-\Gamma_{1} \partial \rho / \partial r\right)}$

with $\Gamma_{1}=\Gamma_{1}\left(P, \rho, X_{\mathrm{m}}\right)$ given by the equation of state this gives $\Gamma_{1}$ along the adiabatic surfaces $r=r_{\mathrm{m}} h(\theta)$. The values of $\Gamma_{1}$ can then be transferred to the mesh $\left(r_{i}, \theta_{j}\right)$ or to the characteristic surfaces $r=r_{\mathrm{m}} g(\theta)$ by cubic interpolation.

This method is not satisfactory in convective zones where the whole region is very nearly adiabatic but, since the composition will be constant in such zones, $\Gamma_{1}$ can be computed directly from the known distribution of $P$ and $\rho$ and the known constant value of $X$.

However, in the fully ionised interior of most stars where, due to evolution, the values of $X$ differ from the initial value $X_{0}$, the values of $\Gamma_{1}$ are only weakly dependent on $X$. In the outer layers, where $\Gamma_{1}$ can vary considerably, the abundance $X$ is unchanged from its initial value $X_{0}$. Given the uncertainties attached to modelling the chemical evolution in rotating stars one could determine approximate values of $\Gamma_{1}$ by taking $X$ constant on characteristic surfaces $(P=$ const. $)$, thus avoiding the calculation described in the preceding paragraphs. This will yield acoustic models suitable for studying the effects of rapid rotation on stellar oscillations.

\section{An example, $M=2 M_{\odot}, X_{c}=0.35, \Omega=\Omega(r)$}

As an illustrative example we take $\Omega=\Omega_{\mathrm{s}} \omega(x)$ as a function only of radius $x=r / R_{0}$, where $\omega(x)$, shown in Fig. 1, decreases from a value of 3 in a central core to 1 in the outer envelope, and has continuous derivatives. As mentioned in Sect. 3.5, with $\Omega$ constant in the outer layers the solution is well behaved at the surface. For the spherically averaged model we took a star of $M=2 M_{\odot}$ with an initial composition $X=0.72, Z=0.02$, evolved to the stage where $X_{\mathrm{c}}=0.35$. The average centrifugal force $2 \Omega^{2} r / 3$ was added to the hydrostatic equation, the remaining equations being those of spherical star. The dimensionless angular velocity $\omega(x)$ was taken as fixed throughout the evolution and $\Omega_{\mathrm{s}}$ determined by requiring conservation of angular momentum for the star as a whole. The initial model had radius $R \approx 2.5 R_{\odot}$ and $\Omega_{\mathrm{s}}=4.3 \times 10^{-5} \mathrm{rad} \mathrm{s}^{-1}$ corresponding to an equatorial velocity of $\approx 80 \mathrm{~km} \mathrm{~s}^{-1}$. The star was evolved to a central hydrogen abundance $X_{\mathrm{c}}=0.35$, at which stage $\Omega_{\mathrm{s}}=1.296 \times 10^{-4} \mathrm{rad} \mathrm{s}^{-1}$. The evolutionary track in the H-R diagram is shown in Fig. 2, where we also show the

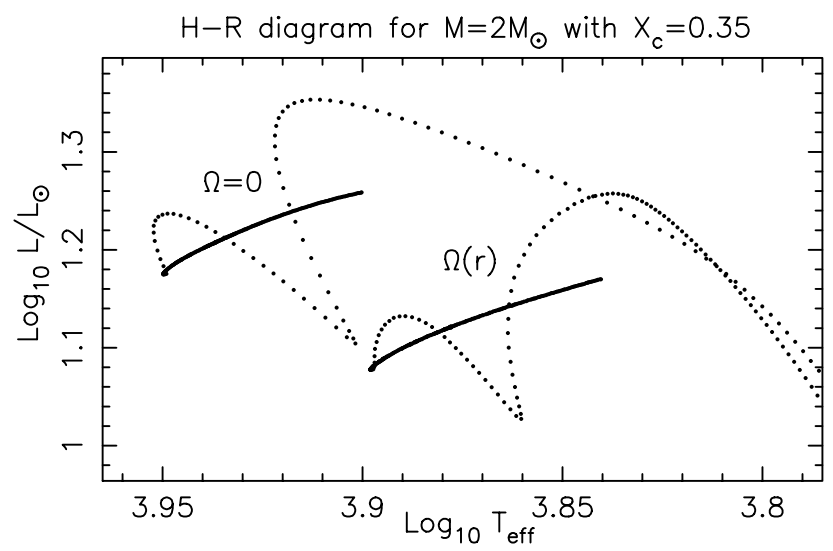

Fig. 2. HR diagram for $M=2 M_{\odot}$ star with $\Omega=\Omega(r)$ and with $\Omega=0$. The initial conditions were $X=0.72, Z=0.02, R=2.5 R_{\odot}, \Omega_{\mathrm{s}}=$ $4.3 \times 10^{-5} \mathrm{rad} \mathrm{s}^{-1}$.

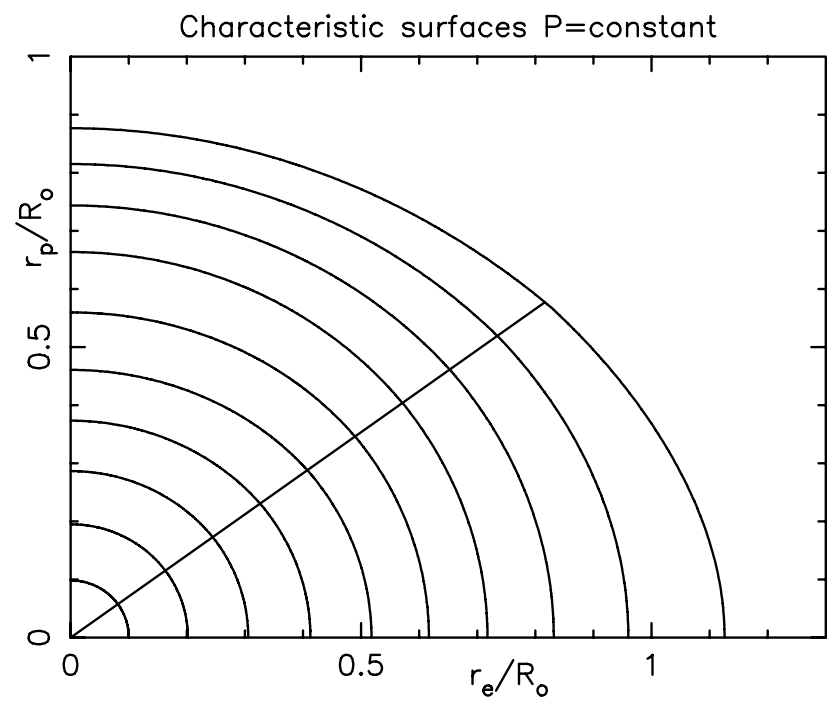

Fig. 3. Characteristic surfaces ( $P=$ const.) in the 2-dimensional model for $x=r / R_{\mathrm{o}}=0,1$ in steps of 0.1 along the fitting angle $\theta_{\mathrm{m}}=$ $\cos ^{-1}(1 / \sqrt{3})$ denoted by the radial line.

evolutionary track for a non-rotating star with the same initial conditions. Note the large effect of rapid rotation on the evolutionary tracks.

The models were produced using the starox evolution code (cf. Roxburgh 2005) with the OPAL2001 equation of state (Rogers \& Nayfonov 2002), OPAL GN93 (Iglesias \& Rogers 1996) and Alexander \& Ferguson (1994) opacities, NACRE (Angulo et al. 1999) nuclear reaction rates, and a radial mesh in mass with $N=2000$ points.

The spherically averaged model was taken to represent the 2-dimensional model along the angle $\theta_{\mathrm{m}}=\cos ^{-1}(1 / \sqrt{3})$, and the procedure described in Sect. 3 followed to produce the 2-d model. The angular mesh had $N_{j}=240$ points and the mesh for solving Poisson's equation had $N_{k}=8$ points. The characteristic surfaces ( $P=$ const.) are shown in Fig. 3 , the ratio of equatorial to polar radius of the model $R_{\mathrm{e}} / R_{\mathrm{p}}=1.2841$, the equatorial rotational velocity $V_{\mathrm{e}}=272.3 \mathrm{~km} \mathrm{~s}^{-1}$, and the ratio of centrifugal force to gravity at the surface equator $\Omega_{\mathrm{s}}^{2} R_{\mathrm{e}}^{3} / G M=0.587$.

In Fig. 4 we show the variation of density along selected characteristics which pass through the points with $r / R_{\mathrm{O}}=$ $0.4,0.5,0.6,0.7$ along the fitting angle $\theta_{\mathrm{m}} . \delta \rho / \rho=0$ for $\theta>\theta_{\mathrm{m}}$ along the characteristic through $r / R_{\mathrm{o}}=0.7$ since this part of the 


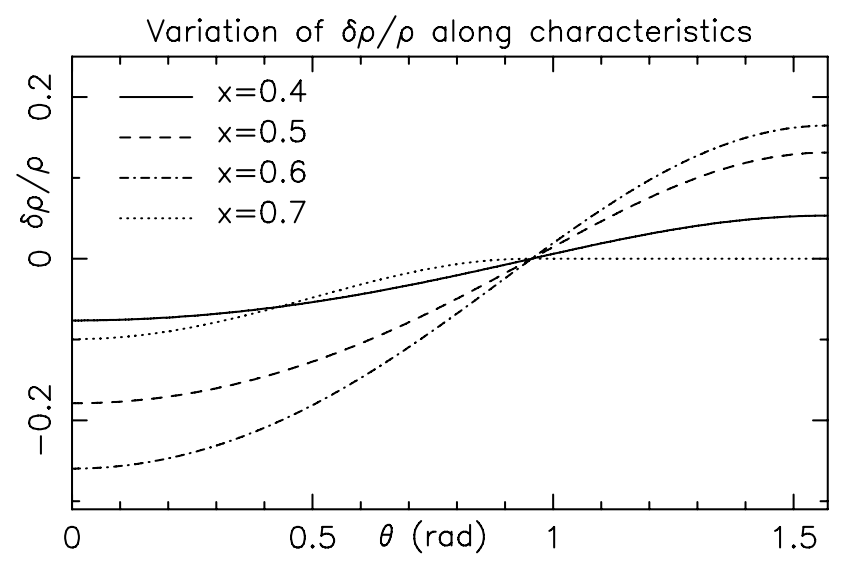

Fig. 4. Variation of $\delta \rho / \rho$ along the characteristics through $x=r / R_{\mathrm{o}}=$ 0.4, 0.5.0, 6, 0.7 along the fitting angle $\theta_{\mathrm{m}}$.

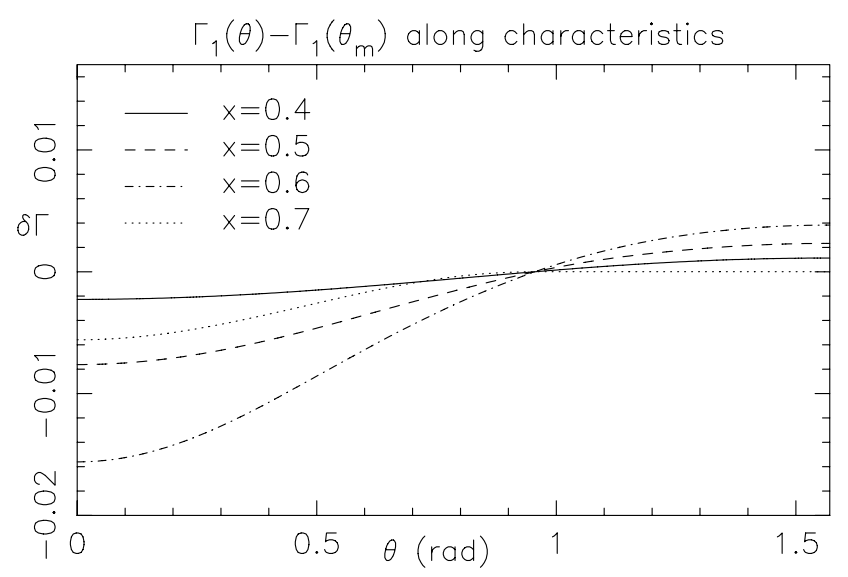

Fig. 5. Variation of the $\Gamma_{1}$ along the characteristics through $x=r / R_{\mathrm{o}}=$ 0.4, 0.5.0, 6, 0.7 along the fitting angle $\theta_{\mathrm{m}}$.

characteristic is in the uniformly rotating region where $\rho, P$ are constant along total potential surfaces $\Psi=\Phi-\Omega^{2} \varpi^{2} / 2=$ const. The other characteristics are in the differentially rotating region where $\rho$ is not constant on surfaces $P=$ const. and $\delta \rho / \rho$ varies by up to $25 \%$, the maximum variation being on the characteristic through $x=0.6$.

In Fig. 5 we show the variation of $\Gamma_{1}$ along these same charactersistics. $\delta \Gamma_{1}=0$ along the characteristic with $r / R_{0}=0.7$ for $\theta>\theta_{\mathrm{m}}$ since this is in the uniformly rotating zone where both $\rho$ and $X$ are constant on surfaces $P=$ const. $\rho$ is not constant on the other characteristics so neither is $\Gamma_{1}$. Again the largest variation is on the characteristic through $x=0.6$.

The mass of the spherically averaged input model was $2 M_{\odot}$. As pointed out above the mass of the rapidly rotating model is slightly changed from this due to the two dimensional redistribution of density; the mass of the $2-\mathrm{d}$ model being $=1.9995 \mathrm{M}_{\odot}$.

\section{Discussion}

The aim of this paper was to show how one can construct moderately realistic hydrostatic and acoustic 2-dimensional models of rapidly rotating evolved stars with any $\Omega(r, \theta)$, given the run of density $\rho_{\mathrm{m}}(r)$ and hydrogen profile $X_{\mathrm{m}}(r)$ along any one angle $\theta_{\mathrm{m}}(r)$, given by some spherically averaged model of evolved rotating stars. It should be noted that in calculating the hydrostatic structure we do not require that the star be in thermal equilibrium; the oscillation properties of a star do not depend on whether or not the star is in thermal equilibrium, only that it be in hydrostatic equilibrium with known $\Gamma_{1}$. The thermal equilibrium of a rotating star depends on the contribution from the transport of energy by circulation currents and by weak turbulence generated by instabilities driven by the circulation and differential rotation. There is considerable uncertainty in modelling these processes and further work is needed in this area (see e.g. Mathis \& Zahn 2004, 2005). The assumptions that go into calculating the structure of the 1-dimensional spherically averaged model are simply carried over into the 2-dimensional models.

The model calculated here takes the $1-\mathrm{d}$ spherically averaged model to represent the 2-dimensional model along the angle $\theta_{\mathrm{m}}=\cos ^{-1}(1 / \sqrt{3})$. For shellular rotation $\Omega=\Omega(r)$ this is what is assumed in the 1-d models when adding the averaged centrifugal force, $2 \Omega^{2} r / 3$, to gravity in the equation of hydrostatic support. It also is the case for slow rotation where, with $\Omega(r)$, all variables $V$ can be expressed as $V(r, \theta)=V_{0}(r)+V_{2}(r) P_{2}(\cos \theta)$ where $P_{2}$ is the second Legendre polynomial. The average over a sphere is then the value at the root of $P_{2}(\cos \theta)$, namely $\theta_{\mathrm{m}}=$ $\cos ^{-1}(1 / \sqrt{3})$. For a general rotation law $\Omega=\Omega(r, \theta)$ this is no longer the case. The mean value of $\Omega^{2} r^{2} \sin ^{2} \theta$ averaged over a sphere is not equal $2 \Omega^{2} r / 3$ and the angle $\theta_{\mathrm{m}}$ along which the $1-\mathrm{d}$ model represents the spherically averaged model depends on the particular $\Omega(r, \theta)$. Such more general 1-d spherically averaged models have yet to be calculated.

However we can still construct consistent 2-dimensional acoustic models that can be used to investigate the oscillation properties of stars with arbitrary $\Omega(r, \theta)$. To construct such models all we require is the distribution of $\Omega(r, \theta)$ and the run of $\rho(r), X(r)$ along any one angle $\theta_{n}$, these do not even need to be the output of a particular 1-d model of the evolution of rotating stars, nor does $\theta_{n}$ have to be the angle corresponding to a spherical average. Models with given $\rho(r), X(r)$ constructed with different fitting angles $\theta_{n}$ will differ, but they will nevertheless all be self consistent $2-\mathrm{d}$ acoustic models. This is important since it provides a recipe for generating a wide range of acoustic models that can be used to study the effects of rotation and internal structure on the oscillation properties of stars, and hence to develop diagnostic tools for probing the rotation and structure of stars using observational data on frequencies obtained from ground and space experiments.

For example, were we to take the spherically averaged model with $\Omega=\Omega(r)$ as being the 2-d model along the pole $(\theta=0)$, our algorithm would still yield a fully self consistent $2-d$ acoustic model. It would not be the same model as that obtained by taking the fitting angle as $\cos ^{-1}(1 / \sqrt{3})$, but it would still be a consistent acoustic model.

If the angular velocity $\Omega=$ const. throughout the star the algorithm presented here reduces to that for uniform rotation presented in Paper I (Roxburgh 2004). The characteristics are the surfaces $P=$ const., from Eq. (7) $\rho$ is constant on these surfaces, which are therefore total potential surfaces $\Psi=\Phi-\Omega^{2} \varpi^{2} / 2=$ const. Eq. (8) gives the shape of these surfaces, the algorithm for determing $\Phi$ in Sect. 3.2 is the same as that in Paper I, and Eq. (17) for $P$ is equivalent to $\mathrm{d} P / \mathrm{d} \Psi=-\rho$. However in Paper I we calculated the full 2-dimensional structure of the rotating star which gave the solution along any angle not just along the angle $\theta_{\mathrm{m}}=\cos ^{-1}(1 / \sqrt{3})$. Thus we can take as input to the algorithm presented in this paper the values of $\rho\left(r, \theta_{n}\right)$ at any angle $\theta_{n}$; the solutions obtained for different $\theta_{n}$ should be, and are, all the same, since the input model is the solution of 2-d equations. 
From the results given in Paper I we can test the accuracy of taking a 1-d spherically averaged model along $\theta_{\mathrm{m}}=\cos ^{-1}(1 / \sqrt{3})$. In Table 6 of Paper I the last entry for $N_{k}=1$ corresponds to the spherically averaged approximation with any variable $V(r, \theta)=V_{0}(r)+V_{2}(r) P_{2}(\cos \theta)$. For uniform rotation the error in using the spherical average approximation is $\leq 10^{-4}$ for $\Omega \leq 0.83 \Omega_{\text {crit }}$ where $\Omega_{\text {crit }}$ is the maximum permitted value of $\Omega$ when centrifugal force balances gravity at the equator.

The example given in this paper has the angular velocity varying only with radius $\Omega=\Omega(r)$; this makes the calculation relative simple. But it can be argued that a more realistic model would have the angular velocity constant along adiabats $S=$ const. and therefore $\Omega=\Omega(S)$; since the adiabats are not spheres this implies $\Omega=\Omega(r, \theta)$. The method of constructing acoustic models described here is for a general, but prescribed, distribution $\Omega(r, \theta)$; to generate models with $\Omega=\Omega(S)$ requires another level of iteration for the distribution of $\Omega$. One can see how such models could be produced. Starting with the same input of $\rho_{\mathrm{m}}(r), \Omega(r), X_{\mathrm{m}}(r)$ along a specified angle $\theta_{\mathrm{m}}$, one constructs a 2-d model as described above. This gives the $P(r, \theta), \rho(r, \theta), X(r, \theta)$ and the surfaces of constant entropy (or adiabats). Taking $\Omega$ constant along adiabats gives a new distribution of $\Omega(r, \theta)$ with which a new model is determined. This process is then iterated to convergence. Implementation of this procedure is the subject of future investigations.
Acknowledgements. This work was supported in part by the UK Particle Physics and Astronomy Research Council under grants PPA/G/S1997/00338 and PPA/G/S/2003/00137.

\section{References}

Alexander, D. R., \& Ferguson, J. W. 1994, ApJ, 437, 891

Angulo, C., Arnould, M., Rayet, M., et al. 1999, A Compilation of Charged Particle Induced Thermonuclear Reaction Rates, NuPhA, 656, 3

Baglin, A., Auvergne, M., Barge, P., et al. 2002, Stellar Structure and Habitable Planet Finding, ed. F. Favata, I. Roxburgh, \& D. Galadi, ESA SP-485, 17 Faulkner, J., Roxburgh, I. W., \& Strittmatter, P. A. 1968, ApJ, 151, 203

Iglesias, C. A., \& Rogers, F. J. 1996, ApJ, 464, 943

Lignières, F., \& Rieutord, M., Reese, D. 2006, A\&A, in press

Maeder, A. 2003, A\&A, 399, 263

Mathis, S., \& Zahn, J.-P. 2004, A\&A, 425, 229

Mathis, S., \& Zahn, J.-P. 2005, A\&A, 440, 653

Osaki, Y. 1966, Pub. Ast. Soc. Jpn., 18, 70

Rogers, F. J., \& Nayfonov, A. 2002, ApJ, 576, 1064

Reese, D., Lignières, F., \& Rieutord, M. 2006, A\&A, in press

Roxburgh, I. W. 1964, MNRAS, 128, 157

Roxburgh, I. W. 2001, A\&A, 377, 688

Roxburgh, I. W. 2004, A\&A, 428, 171

Roxburgh, I. W. 2005, http://www.astro.up.pt/corot/ntools/ tools.html

Roxburgh, I. W., \& Vorontsov, S. V. 2003, Ap\&SS, 284, 187

Zahn, J.-P. 1992, A\&A, 265, 115 